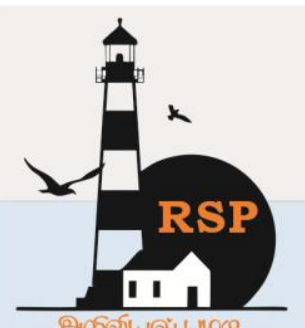

\title{
Effect of Heat Input on the Microstructure and Mechanical Properties of Gas Tungsten Arc Welded AISI 316 Stainless Steel Joints
}

B Karthick ${ }^{1}$, L.Shrihari ${ }^{2}$, M Sakthivel ${ }^{3,}$ S Shriram $^{4}$, V Silambarasan ${ }^{5}$

${ }^{1}$ Assistant Professor, Department of Mechanical Engineering, SNS College of Technology, Coimbatore-

641035, Tamilnadu, India.

${ }^{2,3,4,5}$ Student, Department of Mechanical Engineering, SNS College of Technology, Coimbatore-641035,

Tamilnadu, India.

karthickb.shelan@gmail.com ${ }^{1}$,shriharimech18@gmail.com ${ }^{2}$

\section{Abstract}

For every manufacturing industry, materials that are applicable for particular conditions and are of low economy are highly required. Hence various studies are carried out to determine the working conditions for several materials. The wide application of steel in industry demands a significance study on physical, chemical and mechanical properties of steel, Here, the characteristic changes in the mechanical and microstructure properties due to the effect of heat inputs of gas tungsten arc welded AISI 316 Stainless steel were examined. The major issue faced during the welding process of stainless steel is the increase in grain growth in the heat affected zone (HAZ). The welding process have been carried out using three heat input parameters on the AISI 316 Stainless steel from the operating window of the gas tungsten arc welding process $(G T A W)$ and they are categorized as low heat $(2.563 \mathrm{KJ} / \mathrm{mm})$, medium heat $(2.784 \mathrm{~kJ} / \mathrm{mm})$ and high heat $(3.017 \mathrm{~kJ} / \mathrm{mm})$. These welded joints of AISI 316 SS have been subjected to evaluation of tensile testing microstructure and mechanical properties so as to interrupt and infer the impact of thermal arc energy on the mechanical properties and microstructure of AISI 316 SS welded joints. From this study, it has been inferred that the heat input has notable effect on Ultimate Tensile Strength, Hardness and Microstructure on AISI 316 SS GTAW plates.

Keywords: AISI 316 Stainless steel, Heat affected zone (HAZ), Gas tungsten arc welding process (GTAW)

\section{Introduction}

Stainless steels are widely used in many manufacturing industries. The Stainless steels are mainly categorized into austenitic, ferritic, martensitic and duplex. Austenitic steels are the steels which have the primary phase as austenite they are generally face centered cubic crystal (FCC). Hardening of Austenitic steel using normal heat treatment is difficult. Ferritic steels are the steels which have the primary phase as ferrite they are generally body centered cubic crystal (BCC), they are generally less ductile when compared to austenitic steel and it also cannot be hardened by heat treatment. The microstructure of martensite was first discovered by German, metallurgist named Adolf Martens around the year 1890, Quenching and cooling below 450 degrees can transform martensite into austenite steel. The major composition of SS is chromium and nickel 


\section{www.rspsciencehub.com}

due to the presence of chromium it makes SS a corrosion resistant metal. The main concept of this project is to introduce the heat to the metal surface and due to raise in the heat input the properties of the metal will change automatically. This heat input can be given to the metal by different types of modes. The heat input can be changed by altering the current with respect to voltage. The voltage and current are noted down and the heat input can be found by calculating in formula. After applying heat on the metal surface the specimen in prepared according to the ASTM standard norms for the Mechanical characterization and the specimen is machined to the required size as it should contain (HAZ1, HAZ2, Parent material) and the microstructural studies were carried out, The changes on the microstructure and mechanical properties of the metal are revealed and the results are analyzed and inferred. In the present world every manufacturing industry has a metallurgical division in order to make feasible study on the metals and materials which are used in the production division in a as the little deviation in microstructure and mechanical properties may have a huge impact in the final outlet product. This project helps to analyze the metals which are used various climatic conditions and their variation in the mechanical properties like tensile strength, compression and hardness of the metal used This project also helps to reveal the variation in the microstructure of the metal due to its climatic conditions. The properties like ductility, brittleness, malleability can be visualized with the help of the microstructural studies revealing the dendrites formation and grain formation in the metal which helps to analyze about the metal and information about suitable application of the metal and helps in gaining the better results for those applications.[1-4].

\section{Experimental details}

\subsection{Base and filler material combination}

The AISI 316 SS plates of dimensions $200 \mathrm{~mm}$ $\mathrm{X} 100 \mathrm{~mm}$ X $6 \mathrm{~mm}$ were taken as base material which were cut from a rolled sheet and 316 SS solid electrode of $3.15 \mathrm{~mm}$ diameter were taken as filler material. Table $1 \& 2$ represents the chemical composition of the base and the filler metal used

Volume 02 Issue 08 August 2020

Table.1.Chemical Composition of SS316

\begin{tabular}{|c|c|}
\hline Element & Chemical Composition of SS316 \\
\hline $\mathrm{Cr}$ & 18 \\
\hline $\mathrm{Ni}$ & 10 \\
\hline $\mathrm{Mn}$ & 2 \\
\hline $\mathrm{Si}$ & 0.75 \\
\hline $\mathrm{Mo}$ & 1.66 \\
\hline $\mathrm{C}$ & 0.08 \\
\hline $\mathrm{N}$ & 0.10 \\
\hline $\mathrm{S}$ & 0.03 \\
\hline $\mathrm{P}$ & 0.045 \\
\hline $\mathrm{Fe}$ & Remainder \\
\hline
\end{tabular}

Table.2. Chemical Composition of SS316L

\begin{tabular}{|c|c|}
\hline Element & $\begin{array}{c}\text { Chemical Composition of } \\
\text { SS316L }\end{array}$ \\
\hline $\mathrm{Cr}$ & 17.6 \\
\hline $\mathrm{Ni}$ & 10.12 \\
\hline $\mathrm{Mn}$ & 1.68 \\
\hline $\mathrm{Si}$ & 0.064 \\
\hline $\mathrm{Mo}$ & 2.38 \\
\hline $\mathrm{C}$ & 0.025 \\
\hline $\mathrm{N}$ & 0.0675 \\
\hline $\mathrm{S}$ & 0.003 \\
\hline $\mathrm{P}$ & 0.025 \\
\hline $\mathrm{Ti}$ & 0.001 \\
\hline $\mathrm{Nb}$ & 0.045 \\
\hline $\mathrm{Fe}$ & Balance \\
\hline
\end{tabular}




\section{Welding Procedure}

The first step to be followed before welding is to clean the work material mechanically and chemically as well in ways to avoid dust particles and other effluents that may creep into the weld and possibly result into welds defects. In order to ensure full penetration, in this work double V-groove was used, the groove angle was taken according to the standard norms. After the tacking process of the plates the welding was carried out using GTAW process with the welding parameters as mentioned below before giving the second pass an interpass temperature of around 150C was maintained. Despite GTAW process carried out by using manual mode, but during recording of time utmost care was ensured as to carry out calculations of welding speed for heat input calculations. In this present work the best welding practice available in the fabrication industry was used. It is an established fact that welding current is the most influential variable in the arc welding process as it affects the melting rate of base and filler material and it also plays a major role in disturbing the current density. So considering this fact three different heat input combinations were chosen corresponding to different welding current i.e. $120 \mathrm{~A}$ (low heat input), $150 \mathrm{~A}$ (medium heat input) and $180 \mathrm{~A}$ (high heat input) were selected for the present work. There are two primary reasons for using the before mentioned current values, arc energies which are sufficient to cause adequate fusion of the base and weld metal can be obtained by this spectrum of heat input combinations and to cause an direct and significant influence on the microstructure and tensile properties of the weld metal, the slight increase of $30 \mathrm{~A}$ current combination is much sufficient as anticipated.Visual inspection of the joints before and after welding was done so as to ensure that all weld beads are possessed with good geometrical consistency and are free from visible defects in surface such as blow holes and surface porosity, the non-consumable used for the joints in this present work is tungsten electrode $=\mathrm{EW}-\mathrm{Th}-2$ (Thoriated tungsten) of $3 \mathrm{~mm}$ diameter, the Shielding gas used is industrially pure argon with flow rate of $15 \mathrm{~L} / \mathrm{min}$, Electrode to work angle was maintained at 45degree the Polarity was maintained as DC electrode positive.

\section{Specimen sampling}

The samples for mechanical investigation like tensile testing, micro hardness testing and microstructural studies were taken from the weld pads as schematically illustrated.

\subsection{Tensile test}

The specimen of each heat input combinations was machined out from the weld pads, each specimen for tensile testing was prepared in accordance to ASTM E08 standards [8] in simple words the samples were machined to dog bone shaped as illustrated schematically in Fig 1 .

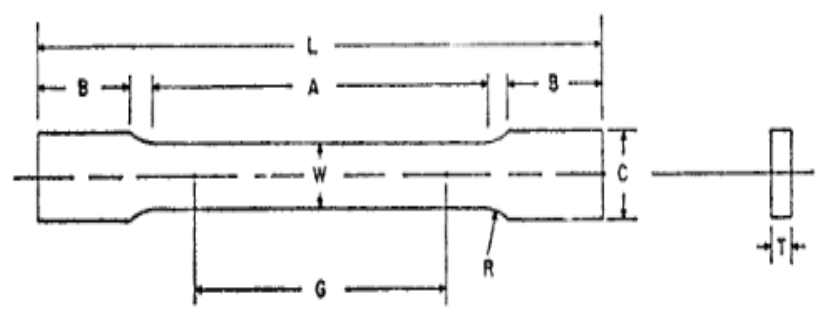

Fig 1: Tensile testing Specimen

\section{Results and Discussions:}

\subsection{Result of Tensile Test:}

The transverse tensile strength of all beads made using different heat input conditions has been evaluated. Totally six specimens were tested under same atmospheric conditions and the tensile test result so obtained show that maximum tensile strength of $637.5 \mathrm{~N} / \mathrm{mm}^{2}$ is possessed by the specimen made using medium heat input combination followed by tensile strength of 609.1 $\mathrm{N} / \mathrm{mm}^{2}$ is possessed by the specimen made using low heat input combinations. Inference obtained from the results is that in normal condition the tensile strength is found to be $495 \mathrm{~N} / \mathrm{mm}^{2}$ and in heat input condition the tensile strength is $637.5 \mathrm{~N} / \mathrm{mm}^{2}$

\subsection{Results of Vickers Hardness Test:}

The hardness strength of all beads made using different heat input conditions has been evaluated. In each condition six specimens were tested and the corresponding Vickers hardness number (VHN) is found. It can be seen that the hardness number is high for parent metal region and reduces gradually towards the Heat Affected Zone and the Weld Metal regions. No external 
cooling was done during welding process. Hence cooling was done by atmospheric air. This result in slow cooling rate of the HAZ and Weld metal regions compared to the cooling rate of the parent metal.

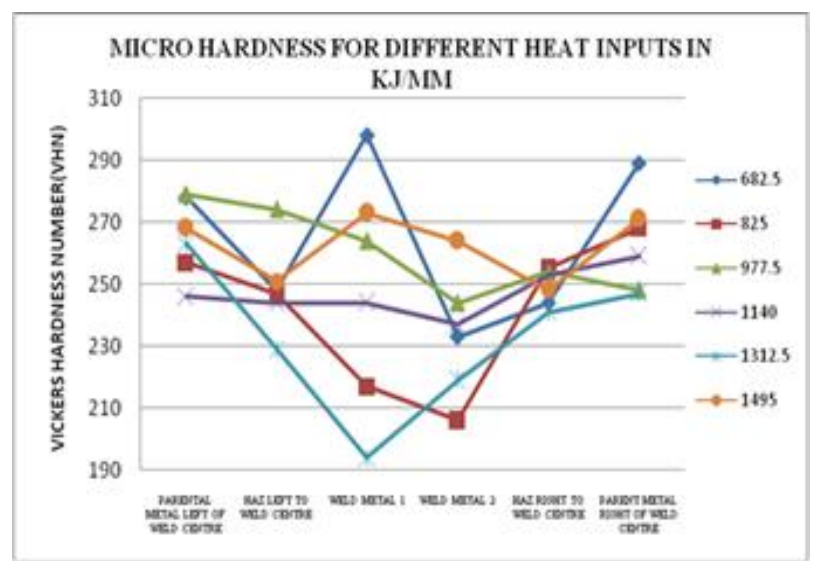

Chart.1.Vickers hardness test

\subsection{Results of Microstructure Study:}

The Microscopic Examination of the six welded specimens were done using Optical microscope. The Microscopic images show that the material consists of Austenite and a small amount of Delta ferrite after the welding process. The areas of HAZ and Weld metal exhibit delta ferrite due to heat treatment. At Low heat input, the formation of delta ferrite initiates as it can be seen clearly in the Microscopic images of the Weld specimen shown in Fig $3 \& 4$. As the heat input increases, the delta ferrite becomes more and more dense and at a certain heat input of 1140 $\mathrm{KJ} / \mathrm{mm}$, a fine grain structure of delta ferrite can be found in the metallographic images. Delta ferrite content can be easily calculated using Fig 2 . Schaffler diagram. Both the diagrams use nickel equivalent and chromium equivalent to determine the delta ferrite content in stainless steels.

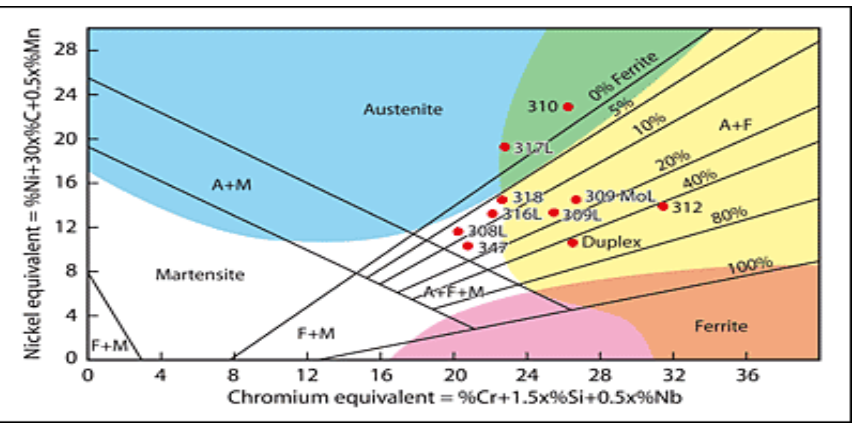

Fig.2. Schaffler Diagram
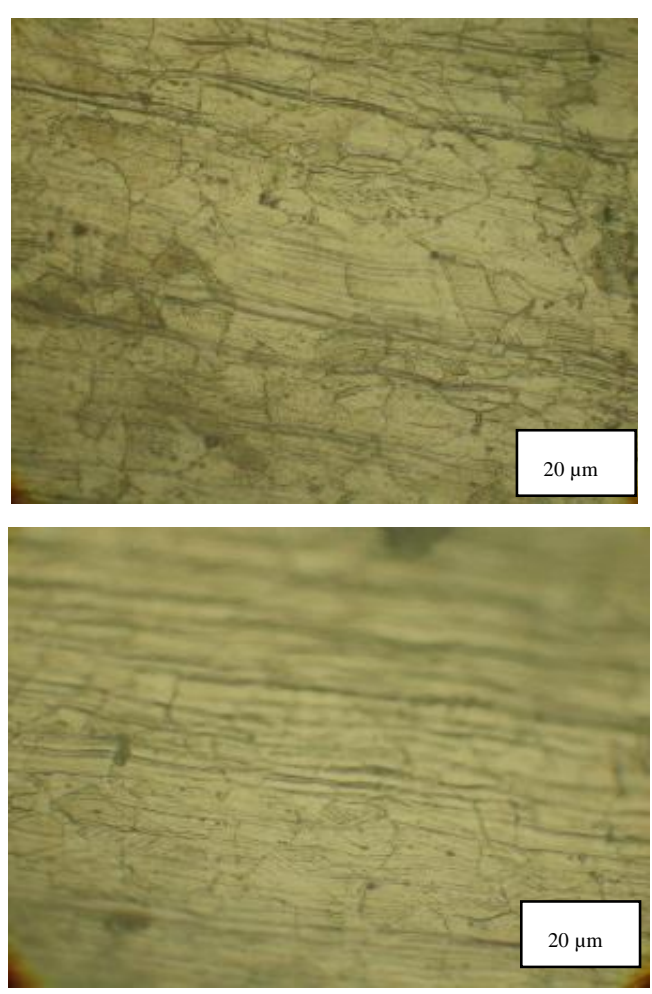

Fig 3: Microscopic Images of the Parental Region of Specimens at $20 \mu \mathrm{m}$
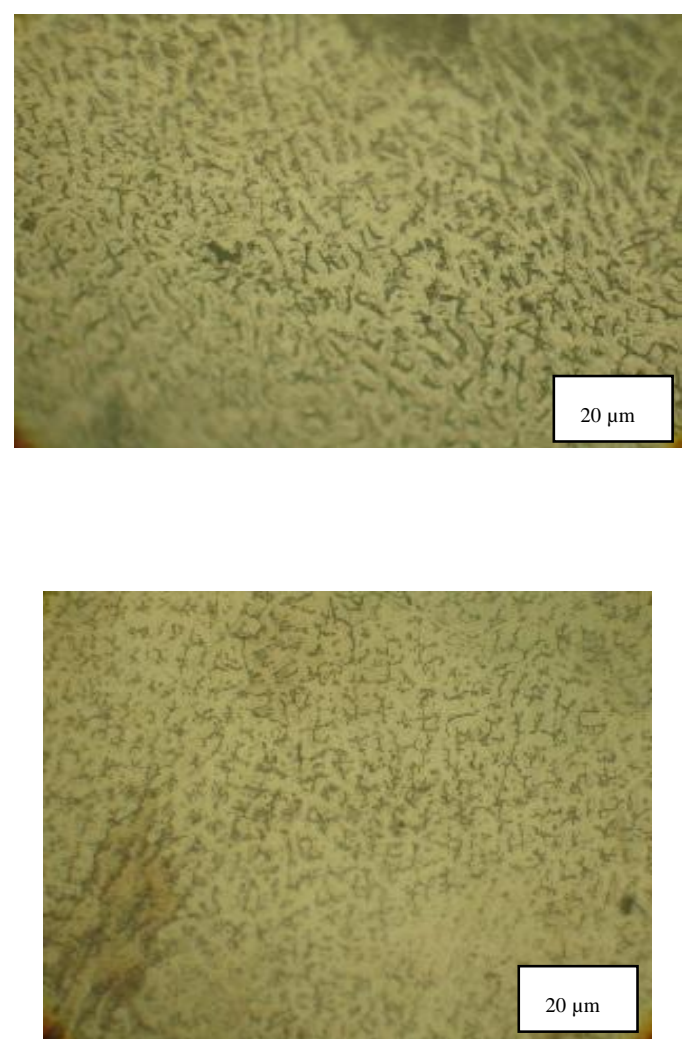

Fig 4: Microscopic Images of the Weld Region Specimens at $20 \mu \mathrm{m}$ 


\section{Conclusions}

- The cooling rate seems to have significant effect on tensile property of the weld. The failure in the parent material infers that the grain size is finer at the fused region.

- The Weld metal regions are found to possess low hardness than the HAZ regions and HAZ regions have less hardness than the parent metal regions.

- From the graph, it is known that the hardness values form a ' $\mathrm{V}$ ' shaped curve denoting the decrease in hardness towards the weld region from the parental region.

- Hence the heat input provided had a significant effect on the delta ferrite content leading to increase in delta ferrite content at the weld region.

- From this, the reason for increase in tensile strength at the weld metal is justified causing the material to fail at the parental metal region.

- AISI 316 is known for its immunity to sensitization. Hence the formation of chromium carbides or the formation of secondary phase of delta ferrite (sigma phase) is highly minimized.

\section{References}

\section{Journals}

[1] Subodh Kumar, A.S. Shahi [2011] " Effect of heat input on the microstructure and the mechanical properties of gas tungsten arc welded AISI 304 stainless steel joints" [Materials and Design Vol. 32 (2011) pp. 3617-3623].

[2] O.P.Khanna"Welding Technology" Metalloagraphic and Microstructure studies from the "Metallographic and Microstructures"[2004] by ASM HAND BOOK.

[3] "Dr. Sc. Almaida Gigović-Gekić1, Dr.Sc. Mirsada Oruč2, Dr. Sc. Mirko Gojić3 [2011] "Determination Of The Content Of Delta Ferrite In Austenitic Stainless Steel Nitronic 60" [Materials and technology, Vol. 46 (2012) No. 5, pp. 519-523]. Dekker, C. (1999). Carbon Nanotubes as Molecular Quantum Wires. Physics Today, 52(5), 22.
[4] A. F. PADILHA and P. R. RIOS [2002] "Decomposition of Austenite in Austenitic Stainless Steels" [ISIJ International, Vol. 42 (2002), No. 4, pp. 325-337]. 